\section{Choriocarcinoma with lung metastases during pregnancy with successful delivery and outcome after chemotherapy}

J Nabers, T A W Splinter, H C S Wallenburg, F J W ten Kate, R Oosterom, C Hilvering

\begin{abstract}
A patient with an intrauterine pregnancy of 27 weeks had a coexisting pulmonary metastatic choriocarcinoma. On the chest radiograph the lung metastases appeared as pulmonary infiltrates, simulating atypical pneumonia. Serum human chorionic gonadotrophin levels were normal for gestational age. Treatment with methotrexate was successful. This is the first reported case of choriocarcinoma in a woman with a pregnancy of less than 35 weeks in which both mother and child survived. The case emphasises the need to consider choriocarcinoma in any pregnant woman who presents with haemoptysis and pulmonary nodules or infiltrates.
\end{abstract}

Choriocarcinoma associated with a viable pregnancy is extremely rare. Metastases may be fatal before the diagnosis of choriocarcinoma is even suspected. ${ }^{1}$ We describe a normal pregnancy coexisting with a metastatic choriocarcinoma of the lungs. Serum human chorionic gonadotrophin levels were normal for the period of gestation and the chest radiograph appearance suggested an inflammatory process rather than malignancy. Timely diagnosis of the disease is important for successful treatment and aggressive diagnostic procedures may therefore be warranted.

\section{Case report}

A 36 year old woman with a 27 week pregnancy was admitted complaining of dyspnoea and hemoptysis in May 1987. During 1974-84 she had had seven term pregnancies and two spontaneous abortions. In July 1985 and March 1986 she had had further spontaneous abortions, both after 18 weeks' amenorrhoea. Her periods were then normal until October 1986, when she once again became pregnant. In December 1986 she had an episode of cough and production of green sputum. From the 13 th to the 17th week of pregnancy painless vaginal bleeding occurred. In March 1987 she became progressively more dyspnoeic and she consulted her physician when she developed haemoptysis. On admission she was breathless but not cyanosed, and had bilateral basal inspiratory crackles. There were no signs of infection. Arterial blood gas values were: $\mathrm{pH}$ $7 \cdot 45$, carbon dioxide tension $3.7 \mathrm{kPa}$, oxygen tension $10.6 \mathrm{kPa}$, oxygen saturation $96 \%$. The size of the uterus was appropriate for 28 weeks' gestation. The serum human chorionic gonadotrophin level was $19250 \mathrm{U} / 1$ (Abbott enzyme immunoassay), which was normal for the gestational age (range 5000-50 000 U/1). Chest radiography showed bilateral fluffy, fairly confluent densities simulating pneumonia. Ultrasound examination showed a normal placenta; no pathological abdominal mass was detected. Bronchoscopic inspection showed an oedematous mucosa with traces of blood. Cytological examination of secretions obtained during bronchoscopy suggested malignancy. Open lung biopsy was performed on the fourth day after admission despite her rapidly worsening condition and radiological progression of the alveolar infiltrates (fig 1).

Histological examination showed a high grade malignant tumour with necrotic areas and haemorrhage. The tumour was arranged in solid masses with papillary formations and consisted essentially of two distinctive cell types, one type compatible with syncytiotrophoblasts and another with cytotrophoblasts. Many cells proved to be positive for human chorionic gonadotrophin.

The patient was treated with intravenous methotrexate (fig 2a). Several hours later she had severe bilateral pleuritic pain, which could be suppressed only by high doses of morphine. Treatment was continued according to the protocol of the New England Trophoblastic Disease Center for low risk gestational trophoblastic neoplasms ${ }^{2}$ - that is, methotrexate 1 $\mathrm{mg} / \mathrm{kg}$ intramuscularly every other day for four doses and leucovorin $15 \mathrm{mg}$ orally 30 hours after each dose of methotrexate. The courses are given every other week and are repeated twice after the serum human chorionic gonadotrophin levels have become normal. During treatment the serum human chorionic gonadotrophin levels fell to a plateau at $7000 \mathrm{U} / 1$, which is low for the gestational age. Production of $\beta$ subunits, measured by the IRE-Medgenix radioimmunoassay (normal $<1 \mu \mathrm{g} / \mathrm{l}$, range for gestational age 7-45 $\mu \mathrm{g} / \mathrm{l}$ ), was disproportionally reduced, with a decline in serum $\beta$ human chorionic gonadotrophin levels of $87 \%$; but intact human chorionic gonadotrophin as measured by Hybritech assay (normal $<25 \mathrm{U} /$ 1 , range for gestational age $6000-48000 \mathrm{U} / 1$ ) decreased also (fig $2 b$ ).

After the fourth course of methotrexate the cervix was ripened with local prostaglandin $\mathrm{E}_{2}$ and labour was induced at 34 weeks. A healthy boy of $2000 \mathrm{~g}$ was born. Histological examination of the placenta showed no malignancy. Postpartum serum human chorionic gonadotrophin levels fell to normal ( $<5 \mathrm{U} / \mathrm{l}$ ) according to a log linear pattern (fig $2 a$ ). The chest radiograph became normal. Two years after 
Figure 1 Posteroanterior chest radiograph showing disseminated irregular, fluffy densities, mostly confluent, simulating pneumonia with fading of the heart figure and right diaphragm. There are no signs of cardiac failure, and no mediastinal or hilar masses are seen.
Figure 2 (a) Time course of change in serum human chorionic

gonadotrophin as shown by the Abbott enzyme

immunoassay (Abbott)

$\$ 200 \mathrm{mg}$ methotrexate,

intravenous bolus

injection; $75 \mathrm{mg}$

intramuscular

methotrexate. After

delivery (1) the values

became normal. (b) Time

course of serum human

chorionic gonadotrophin

after a single bolus

injection of methotrexate

(1) on the third

postoperative day,

measured by different

methods. The enzyme

immunoassay from Abbott

( measures

intact $H C G$ and $\beta H C G$

subunits; the Hybritech

enzyme immunoassay

(Hybritech Europe, Liège,

Belgium:

measures only intact

human chorionic

gonadotrophin, wherea

the IRE-Medgenix

radioimmunoassay (IRE-

Medgenix, Fleurus

Belgium A-.-A)

measures predominantly $\beta$

subunits with about $10^{\circ}$ 。

cross reactivity with intact

human chorionic

gonadotrophin.

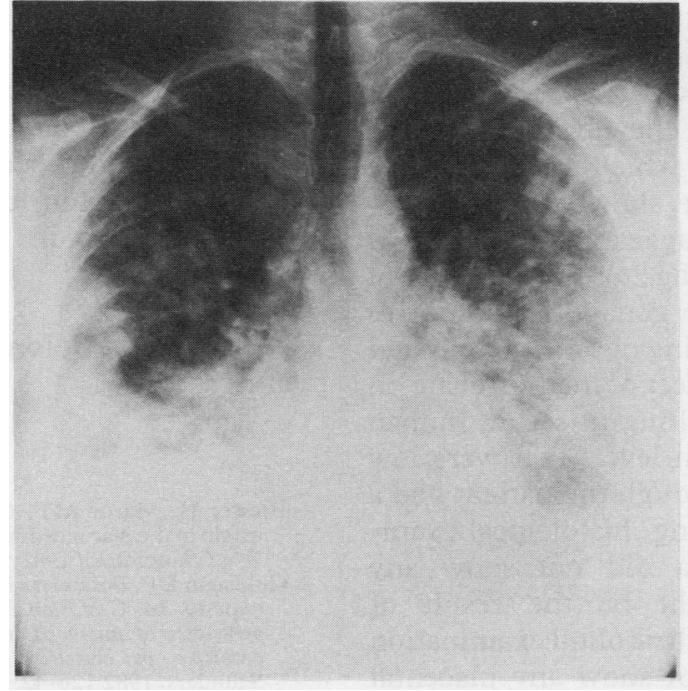

patients have well defined, round nodular lesions; usually less than 10 are present, and solitary nodules have been described. The nodules may cavitate. Secondly, in $5-15 \%$ of cases the radiological appearances have a miliary or alveolar pattern with indefinite borders simulating an inflammatory process. Progression of this type of lesion may be remarkably rapid, as in our patient. Thirdly, embolisation of the pulmonary artery by tumour metastases may cause infarction and pulmonary hypertension; although an incidence of $25^{\circ}{ }_{0}$ has been reported, ${ }^{8}$ current experience suggests a figure of less than $1 \%{ }^{7}$ These patients show no typical radiological appearance, though variable amounts of pulmonary shadowing and evidence of loss of lung volume may occur.

In choriocarcinoma serum human chorionic gonadotrophin levels are usually raised. This is

delivery mother and son are in good health and do not show any evidence of disease.

\section{Discussion}

Choriocarcinoma is a relatively uncommon tumour and its occurrence during pregnancy is even more rare. Kuhn et al $^{3}$ found 28 published cases and added two of their own. The most common symptom is vaginal bleeding. A third of patients present with signs of metastases. Metastases occur frequently in the lungs ( $80 \%$ ) and less often in the vagina $(30 \%)$, pelvis $\left(20^{\circ}{ }_{0}\right)$, and brain $(10 \%){ }^{4}$ Primary choriocarcinoma of the lung ${ }^{5}$ and metastatic endobronchial tumour with bronchial obstruction and pleural effusion ${ }^{6}$ have been described. There are three main types of pulmonary choriocarcinoma, all caused by haematogenous metastases. $^{78}$ Firstly, in $65-95 \%$ of cases

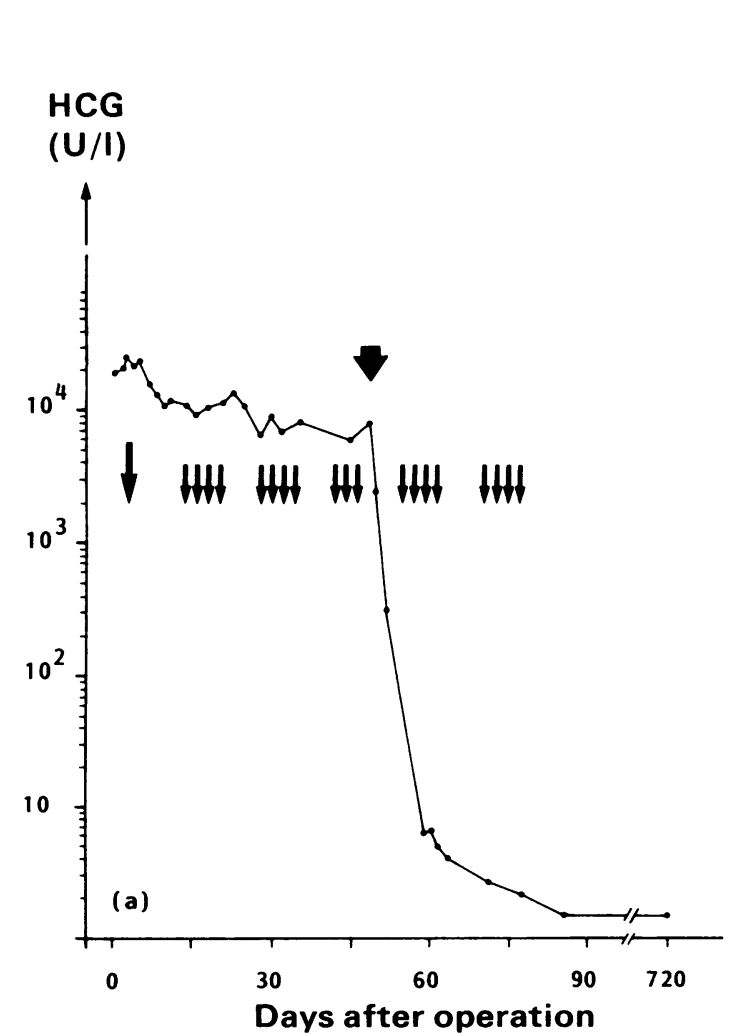
tumours that occur a long time after pregnancy. In the case of choriocarcinoma coexisting with pregnancy, where serum human chorionic gonadotrophin levels have been reported, they have been substantially raised except in one case, where levels were near normal. ${ }^{9}$

In our case histological examination of the lung biopsy specimen showed a tumour densely populated by cells containing human chorionic gonadotrophin. The serum human chorionic gonadotrophin levels were in accordance with the patient's gestational age, suggesting that the tumour mass was small; in that case the extensive lesions on the chest radiograph may represent haemorrhage, or else a tumour secreting little or no human chorionic gonadotrophin.

Treatment with methotrexate evoked a strong reaction, with chest pain probably

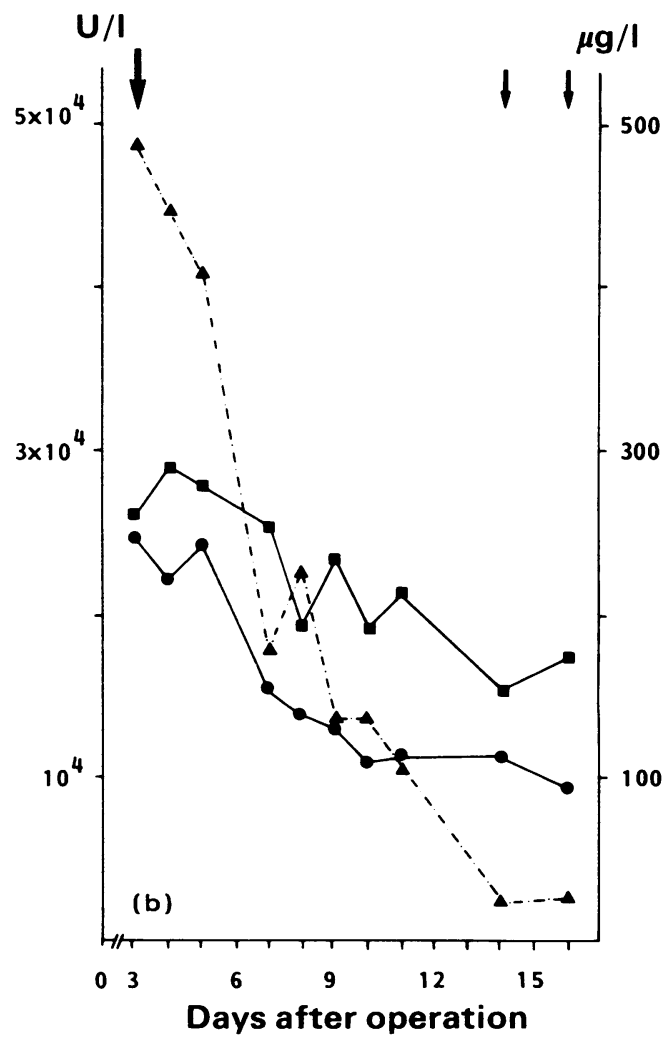


caused by tumour necrosis and pleural irritation. The human chorionic gonadotrophin produced during the plateau phase (fig $2 a$ ) is probably derived from the normal placenta. The titre of $\beta$ subunits, which are likely to be produced by the tumour, showed a disproportionally rapid decline, suggesting that this was due to tumour necrosis (fig 2b). The decrease of intact human chorionic gonadotrophin titres may be attributed to killing of tumour cells and perhaps to a negative effect of methotrexate on placental function, resulting in serum human chorionic gonadotrophin levels that were low for gestational age. Although the patient had a period of vaginal bleeding, histological examination of the placenta did not show any malignancy. This might be the result of chemotherapy, though ultrasound examination before treatment did not show any placental tumour. Spontaneous regression of primary choriocarcinoma has been reported, even when extensive dissemination was present. ${ }^{10}$

To our knowledge, only five cases of gestational choriocarcinoma coexisting with intrauterine pregnancy have been described in which both mother and child survived. ${ }^{11}$ These cases occurred in pregnancies of 35 weeks or more, in which survival of the fetus could be expected. In our patient a choriocarcinoma was diagnosed at 27 weeks. After an aggressive diagnostic procedure adequate treatment was given and mother and child survived without evidence of disease or major toxicity.

Our case confirms that choriocarcinoma may occur in an otherwise normal pregnancy. Pulmonary metastases may present as alveolar infiltrates. Choriocarcinoma should therefore be considered even when a primary tumour is not detectable and when serum human chorionic gonadotrophin levels are normal. Histological confirmation is essential for proper management.

We thank Mrs S Jongenotter for typing the manuscript.

1 Brewer JI, Mazur MT. Gestational choriocarcinoma. Its origin in the placenta during seemingly normal pregnancy. Am J Surg Pathol 1981;5:267-77.

2 Goldstein DP, Berkowitz RS. Chemotherapy protocols and toxicity. In: Gestational trophoblastic neoplasms. Clinical principles of diagnosis and management. Vol 14: Major problems in obstetrics and gynecology. Philadelphia: Saunders, 1982:126-42.

3 Kuhn RJP, Long AR, Fortune DW. Choriocarcinoma coexistent with intrauterine pregnancy. Aust $N Z J$ Obstet Gynaec 1980;20:94-8.

4 Goldstein DP, Berkowitz RS. The management of gestational trophoblastic neoplasms. Cur Prob Obstet Gynecol 1980;4:1-42.

5 Pushchak MJ, Farhi DC. Primary choriocarcinoma of the lung. Arch Pathol Lab Med 1987;111:477-9.

6 McLeod DT. Gestational choriocarcinoma presenting as endobronchial carcinoma. Thorax 1988;43:410-1

7 Libshitz HI, Baber CE, Hammond CB. The pulmonary metastases of choriocarcinoma. Obstet Gynecol 1977;49: 412-6.

8 Bagshawe KD, Garnett ES. Radiological changes in the lungs of patients with trophoblastic tumors. Br J Radiol 1963;36:673-9.

9 Eriksson A, Gezelius C. Metastatic choriocarcinoma during normal pregnancy presenting as thyroid tumor. Arch Gynecol 1984;236:119-22.

10 van der Werf AJM, Broeders GHB, Vooys GP, Mastboom JL. Metastatic choriocarcinoma as a complication of JL. Metastatic choriocarcinoma as a

11 McCue SA, Greene JB. Metastatic cerebral choriocarcinoma during pregnancy. Min Med 1980:164-6. 